\title{
Eficiência de adubos fosfatados associados ao enxofre elementar na cultura do milho ${ }^{1}$
}

\author{
Jucenei Fernando Frandoloso², Maria do Carmo Lana ${ }^{3}$, Silvano Fontaniva ${ }^{4}$, Rodrigo Vianei Czycza
}

\begin{abstract}
RESUMO
A eficiência agronômica dos adubos fosfatados pode ser afetada pelas fontes de fósforo, propriedades do solo, modo de aplicação e espécie vegetal. Com o objetivo de comparar a eficiência do fosfato natural reativo (FNR) e do superfosfato triplo (SFT) associado ao enxofre elementar, aplicados no sulco de plantio na cultura do milho, instalouse um experimento em condições de campo no município de Toledo, Paraná, em um Latossolo Vermelho eutroférrico. O delineamento experimental consistiu de blocos inteiramente casualizados, em esquema fatorial $2 \times 4 \times 2$, sendo duas fontes de fósforo (FNR e SFT), três doses de adubação (100, 200 e $300 \mathrm{~kg} \mathrm{ha}^{-1} \mathrm{de}_{2} \mathrm{P}_{5}$ ) mais uma testemunha para cada fonte, e adição ou não de enxofre elementar ( 0 e $\left.30 \mathrm{~kg} \mathrm{ha}^{-1} \mathrm{de} \mathrm{S}^{\circ}\right)$, aplicados no sulco de plantio em quatro repetições. As parcelas foram constituídas por quatro linhas com $6 \mathrm{~m}$ de comprimento espaçadas $0,9 \mathrm{~m}$, utilizando-se como área útil as duas linhas centrais desprezando-se $0,5 \mathrm{~m}$ das extremidades. A adubação com $\mathrm{N}$ e $\mathrm{K}$ e os tratamentos com $\mathrm{P} \mathrm{e} \mathrm{S}^{\circ}$ foram realizados manualmente no sulco de plantio, sendo posteriormente semeadas com o híbrido Agroceres 8021. O SFT proporcionou maior rendimento de grãos, em comparação com o FNR, no primeiro ano de cultivo. A eficiência agronômica do FNR foi de 43\% e 33\% na presença e ausência de S, respectivamente. O teor de fósforo no tecido foliar foi inferior com o uso do FNR. A dose de $30 \mathrm{~kg} \mathrm{ha}^{-1}$ de $\mathrm{S}$ elementar não interferiu no $\mathrm{pH}$ do solo, mas aumentou a eficiência da adubação fosfatada.
\end{abstract}

Palavras chaves: Fontes de fósforo, eficiência agronômica, Zea mays L.

\section{ABSTRACTS}

\section{Efficiency of phosphate-fertilizers associated with elemental sulfur in corn}

The agronomic efficiency of phosphate fertilizers may be affected by phosphorus sources, soil properties, application methods and plant species. The objective of this work was to compare the efficiency of natural reactive phosphate (NRP) and triple super-phosphate (TSP) associated with elemental sulfur applied in the planting furrow of corn. The experiment was conducted in an Oxisol, in the city of Toledo-PR. The treatments were arranged in a randomized block design, in a factorial scheme $2 \times 4 \times 2$, with two P sources (TSP and FNR), three fertilizer doses (100, 200 and $300 \mathrm{~kg} \mathrm{ha}^{-1}$ $\mathrm{P}_{2} \mathrm{O}_{5}$ ), a control for each source, with or without addition of elemental sulfur ( 0 and $\left.30 \mathrm{~kg} \mathrm{ha}^{-1} \mathrm{~S}\right)$ applied at planting, with four replications. The plots consisted of four rows (6 $\mathrm{m}$ in length) spaced 0,90 meters.

The plot usable area was formed by the center two rows, with $50 \mathrm{~cm}$ on each end of the plot left as border. $\mathrm{N}$ and $\mathrm{K}$ fertilization and the treatments with P and S were done by hand in the furrow and the hybrid Agroceres 8021 was sown

Recebido para publicação em julho de 2009 e aprovado em julho de 2010

${ }^{1}$ Parte da dissertação de mestrado do primeiro autor apresentada a Universidade Estadual do Oeste do Paraná - UNIOESTE - para obtenção do título de mestre em Agronomia.

2 Engenheiro-Agrônomo, Mestre. Doutorando do Programa de Pós-Graduação em Agronomia da Universidade Estadual do Oeste do Paraná, Campus de Marechal Cândido Rondon, Rua Pernambuco 1777, 859600-000, Marechal Cândido Rondon, Paraná, Brasil. juceneiff@hotmail.com.br

${ }^{3}$ Engenheira-Agrônoma, Doutora. Centro de Ciências Agrárias, Universidade Estadual do Oeste do Paraná, Campus de Marechal Cândido Rondon, Rua Pernambuco 1777, 859600000, Marechal Cândido Rondon, Paraná, Brasil. mclana@unioeste.br

${ }^{4}$ Engenheiro-Agrônomo. silvano_fontaniva@yahoo.com.br

Engenheiro-Agrônomo, Mestre. Solos e Nutrição de Plantas, ESALQ/USP, Caixa Postal 9, 13418-970, Piracicaba, São Paulo, Brasil. rodrigo_czycza@yahoo.com.br 
subsequently. TSP provided the highest yields compared with NRP in the first year of cultivation. The agronomic efficiency of the NRP was $43 \%$ and $33 \%$ with and without S, respectively. The phosphorus content in leaf tissue was lower with NRP. The elemental S at $30 \mathrm{~kg} \mathrm{ha}^{-1}$ did not interfere on the soil $\mathrm{pH}$, but the efficiency of the phosphate fertilization was higher with the sulfur application.

Key words: Agronomic efficiency, phosphorus sources, Zea mayz L.

\section{INTRODUÇÃO}

O suprimento inadequado de fósforo nos estádios iniciais de desenvolvimento da cultura do milho acarreta redução no número de espigas por unidade de área e, conseqüentemente, redução na produção final de grãos (Mengel \& Kirkby, 1987).

Devido a baixa disponibilidade deste nutriente no solo, o fertilizante fosfatado é um dos mais aplicados na agricultura brasileira, sendo encontrados diversos adubos fosfatados no comércio, os quais diferem em concentração de fósforo e solubilidade.

O uso de fosfatos naturais reativos no mercado de fertilizantes no Sul do Brasil foi importante nas décadas de 70 e 80, com a importação do fosfato de Gafsa, então denominado hiperfosfato. Segundo Kaminski \& Peruzzo (1997), a oferta de fosfatos naturais como fonte de P para culturas anuais, a preços inferiores, por toneladas, aos dos superfosfatos, tornou-se atrativos no mercado de fertilizantes e aumentou consideravelmente o seu consumo.

Atualmente, as fontes de fosfatos naturais reativos mais comercializadas são: Gafsa, importado da Tunísia, Arad, importado de Israel e Daoui, importado do Marrocos. Segundo legislação vigente, esses produtos devem apresentar 27\% de $\mathrm{P}_{2} \mathrm{O}_{5}$ total e mínimo de 30\% do teor total solúvel em Ácido Cítrico a 2\% na relação 1:100 (Ministério da Agricultura Pecuária e Abastecimento, 2007).

Um dos pontos mais polêmicos no que diz respeito à adubação fosfatada no Brasil é, notadamente, o que trata de fontes e sua eficiência de uso, influenciada pela solubilidade dos fosfatos. A solubilidade do fosfato natural reativo (FNR) é menor do que a do superfosfato triplo (SFT) e isto se deve ao fato de ele ser obtido pelo processo de moagem da rocha apatítica, enquanto o SFT é obtido após tratamento químico da rocha com ácido fosfórico para torná-lo solúvel. Apesar da menor solubilidade, a aplicação de fosfato natural reativo, principalmente a lanço e incorporado no solo, tem proporcionado aumento de rendimento das culturas (Braga et al., 1991; Kaminski \& Peruzzo, 1997; Korndörfer, 1999).

O uso destes fosfatos é mais viável em solos com $\mathrm{pH}$ em água menor que 5,5, pois, à medida que o pH do solo aumenta, a eficiência desses fosfatos diminui (Peruzzo \&
Wiethölter, 2002). A adição de enxofre elementar ao solo proporciona redução do $\mathrm{pH}$, ao ser oxidado por microorganismos (Thiobacillus), facilitando, assim, a solubilidade do FNR (Stamford et al., 2004).

Desta forma, instalou-se um experimento em condições de campo com o objetivo de comparar a eficiência do fosfato natural reativo e do superfosfato triplo, associado ao enxofre elementar aplicados no sulco de plantio na cultura do milho.

\section{MATERIAL E MÉTODOS}

O experimento foi conduzido em condições de campo, utilizando um híbrido simples de milho (Agroceres 8021), de ciclo precoce, o qual foi cultivado na região de Vila Nova, município de Toledo, Estado do Paraná, em um solo classificado como Latossolo Vermelho eutroférrico de textura argilosa (EMBRAPA, 2006). A área escolhida para o experimento encontrava-se sob sistema de plantio direto há dois anos e estava ocupada com a cultura da aveiapreta, a qual foi dessecada 15 dias antes do plantio.

Previamente, com auxílio de um trado holandês, coletaram-se 12 amostras simples de solo na camada de 0-20 cm de profundidade para formar a amostra composta, a qual foi dividida em duas subamostras. Uma delas foi encaminhada ao Laboratório de Química Agrícola e Ambiental da UNIOESTE, Campus de Marechal Cândido Rondon, para determinação das propriedades químicas; a outra foi enviada ao Laboratório de Física do Solo da UNIOESTE, para a determinação das propriedades granulométricas e, de acordo com os resultados obtidos (Tabela 1), foi realizada a recomendação de adubação potássica, que correspondeu à quantidade de $40 \mathrm{~kg} \mathrm{ha}^{-1}$ de $\mathrm{K}_{2} \mathrm{O}$. Para o nitrogênio, foram aplicados $30 \mathrm{~kg} \mathrm{ha}^{-1}$ de $\mathrm{N}$, na semeadura, mais $90 \mathrm{~kg} \mathrm{ha}^{-1}$ de $\mathrm{N}$ em cobertura, quando as plantas apresentavam entre quatro a seis folhas, fornecidos na forma de uréia.

O delineamento experimental consistiu em blocos inteiramente casualizados, com quatro repetições, sendo um fatorial $2 \times 4 \times 2$, ou seja, duas fontes de fósforo (Fosfato natural reativo - Gafsa, com $28 \%$ de $\mathrm{P}_{2} \mathrm{O}_{5}$ total e $9 \%$ solúvel em ácido cítrico 2\%, e superfosfato triplo com $46 \%$ de $\mathrm{P}_{2} \mathrm{O}_{5}$ solúvel em CNA e água), com três doses de fertilizan- 
Tabela 1. Análise química e granulométrica do Latossolo Vermelho eutroférrico na camada de 0-20 cm antes do plantio, do município de Toledo, Paraná

\begin{tabular}{|c|c|c|c|c|c|c|c|c|c|}
\hline $\mathbf{P}$ & P res & $\mathrm{S}$ & M.O & pH $\mathrm{CaCl}_{2}$ & $\mathbf{H}^{+}+\mathbf{A l}^{3+}$ & $\mathrm{Al}^{3+}$ & $\mathbf{K}^{+}$ & $\mathrm{Ca}^{2+}$ & $\mathrm{Mg}^{2+}$ \\
\hline \multicolumn{3}{|c|}{$\mathrm{mg} \mathrm{dm}^{-3}$} & $\mathrm{~g} \mathrm{dm}^{-3}$ & $0,01 \mathrm{~mol} \mathrm{~L}^{-1}$ & & - & $\mathrm{dm}^{-3}$ & & \\
\hline 2,94 & 17,0 & 4,46 & 35,54 & 5,17 & 5,76 & 0,05 & 0,52 & 5,94 & 1,56 \\
\hline SB & CTC & V & m & Argila & & & & & eia \\
\hline \multicolumn{4}{|c|}{$\mathrm{cmol}_{\mathrm{c}} \mathrm{dm}^{-3}-$} & 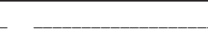 & & $\mathrm{g} \mathrm{k}$ & & & \\
\hline 8,12 & 13,88 & 58,50 & 0,61 & 694,5 & & 142 & & & \\
\hline
\end{tabular}

P e K - extrator Mehlich - l; P res - extraído por resina de troca aniônica; Ca, Mg, Al - extrator KCl $1 \mathrm{~mol} \mathrm{~L}^{-1}$; M.O. - determinado pelo método Walkley \& Black; $\mathrm{H}^{+}+\mathrm{Al}^{3}$ - extraído por solução SMP; $\mathrm{S}$ - extraído com $\mathrm{Ca}\left(\mathrm{H}_{2} \mathrm{PO}_{4}\right)_{2}$, 500mg L ${ }^{-1}$ de $\mathrm{P}$, em HOAc 2 mol L $^{-1}(\mathrm{Alvarez}$ V et al., 2001); análise física pelo método do densímetro (EMBRAPA, 1997).

te (100, 200 e $300 \mathrm{~kg} \mathrm{ha}^{-1}$ de $\left.\mathrm{P}_{2} \mathrm{O}_{5}\right)$, mais uma testemunha para cada fonte, e adição ou não de enxofre elementar (0 e $30 \mathrm{~kg} \mathrm{ha}^{-1}$ de enxofre elementar em pó), totalizando 16 tratamentos e 64 parcelas experimentais.

Cada unidade experimental constituiu-se de quatro linhas de cultivo, cada linha com $6 \mathrm{~m}$ de comprimento e com espaçamento entre linhas de 0,9 m. Como parcela útil, foram utilizadas as duas linhas centrais, reduzidas em 0,5 m nas extremidades, compreendendo uma área útil de $9 \mathrm{~m}^{2}$ por parcela.

A adubação com fósforo, potássio, nitrogênio e enxofre elementar em pó foi realizada manualmente no sulco de semeadura. Para isso, usou-se uma calha de $6 \mathrm{~m}$ de comprimento, onde eram distribuídos os adubos e, posteriormente, depositados no sulco de semeadura. A operação de semeadura do milho foi realizada no dia 29 de setembro de 2005, utilizando semeadora mecânica de quatro linhas, para distribuição apenas das sementes. Aos 25 dias após a semeadura efetuou-se o desbaste para se manter uma população de 60.000 plantas ha-1 . O controle de plantas daninhas, pragas e doenças foram realizadas durante o período de cultivo, conforme necessidade, utilizando-se produtos recomendados para cada situação.

Foram efetuadas amostragens de tecido vegetal no início do florescimento da cultura (80 dias após a semeadura). De cada parcela, foram coletadas folhas de dez plantas bem desenvolvidas e representativas. De acordo com as recomendações de Malavolta et al. (1997), coletou-se a primeira folha abaixo e oposta da espiga. As amostras foram encaminhadas para o Laboratório de Fertilidade do Solo e Nutrição Mineral de Plantas da UNIOESTE, onde foram lavadas e colocadas para secar em estufa de ventilação forçada a $60^{\circ} \mathrm{C}$ por um período de 72 horas, sendo posteriormente moídas e homogeneizadas.

Para determinação dos teores de P e S, as amostras de tecido vegetal foram submetidas à digestão nítricoperclórica na proporção de 3:1. Nos extratos, o teor de P foi determinado por espectrofotometria UV-vis, segundo metodologia de Defelipo \& Ribeiro (1981) e o S dosado por turbidimetria, segundo metodologia de Alvarez V et al. (2001).
Antes da colheita, realizada em fevereiro de 2006, foram coletadas aleatoriamente 10 espigas de cada parcela, para avaliação dos componentes de produção e posterior determinação do teor de P no grão, conforme metodologia usada no tecido vegetal. A estimativa da produtividade foi obtida efetuando-se a colheita da área útil de cada parcela $\left(9 \mathrm{~m}^{2}\right)$, com posterior correção para 13\% de umidade.

Após a colheita, foram coletadas amostras de solo nas profundidades de 0-10 cm e 10-20 cm, utilizando-se um trado holandês, sendo quatro amostras simples, na linha de semeadura dentro de cada parcela, para a formação das amostras compostas. Foram avaliados o pH em cloreto de cálcio $0,01 \mathrm{~mol} \mathrm{~L}^{-1}$; o P, extraído com resina de troca de íons e pelo extrator Mehlich-l e determinado por espectrofotometria UV-vis; e o S, utilizando-se como extrator o fosfato de cálcio em ácido acético $2 \mathrm{~mol} \mathrm{~L}^{-1} \mathrm{e}$ dosado por turbidimetria em espectrofotometria UV-vis, segundo metodologia de Alvarez V et al. (2001).

O índice de eficiência agronômica (IEA), citado por Goedert \& Lobato (1984) e Moreira et al. (2002), foi calculado da seguinte forma: IEA $(\%)=\left[\left(\mathrm{Y}_{2}-\mathrm{Y}_{1}\right) /\left(\mathrm{Y}_{3}-\mathrm{Y}_{1}\right)\right] \mathrm{x}$ 100, onde: $Y_{1}=$ Produtividade de grãos obtidos pela parcela que não teve aplicação de $\mathrm{P} ; \mathrm{Y}_{2}=$ Produtividade de grãos obtidos pela fonte testada (FNR) nas doses de P em estudo; $\mathrm{Y}_{3}=$ Produtividade de grãos obtidos pela fonte de referência (SFT) nas doses em estudo.

Calculou-se ainda a eficiência de utilização do fertilizante (EUF) na presença e ausência de enxofre elementar, utilizando-se a seguinte fórmula, adaptada de Siddiqi \& Glass (1981): EUF = Aumento de produtividade em relação à testemunha $\left(\mathrm{kg} \mathrm{ha}^{-1}\right) /$ doses de $\mathrm{P}_{2} \mathrm{O}_{5}$ aplicadas $\left(\mathrm{kg} \mathrm{ha}^{-1}\right)$.

Os resultados obtidos foram submetidos a análises de variância, de regressão e ao teste de médias. As equações de regressão foram selecionadas, escolhendo-se os modelos significativos com maior coeficiente de determinação $\left(\mathrm{R}^{2}\right)$.

\section{RESULTADOS E DISCUSSÃO}

Na tabela 2, é apresentado o resumo da análise de variância para os teores de P-foliar, S-foliar, P-grão, pro- 
Tabela 2. Resumo da análise de variância para teores de P foliar, S foliar, P grão, produtividade (Produt.), teores de P no solo extraídos por Mehlich-l (M-1) nas profundidades de 0-10 cm (P 10) e 10-20 cm (P 20), P no solo extraído por resina de troca aniônica na profundidade de $0-10 \mathrm{~cm}$ (P 10 res.), teor de $\mathrm{S}$ disponível nas profundidades de 0-10 cm (S 10) e 10-20 cm (S 20), e o pH na profundidade de 0-10 cm em função da aplicação de fosfato natural reativo (FNR), superfosfato triplo (SFT), e adição ou não de enxofre elementar (S)

\begin{tabular}{|c|c|c|c|c|c|c|c|c|c|c|}
\hline $\begin{array}{l}\text { Fontes de } \\
\text { variação }\end{array}$ & P foliar & $S$ foliar & P grão & Produt. & P 10 M-I & P 20 M-I & P 10 res. & S 10 & S 20 & pH CaCl${ }_{2}$ \\
\hline & \multicolumn{10}{|c|}{ - Valores de F - } \\
\hline $\begin{array}{l}\text { Fonte de } \\
\mathrm{P}_{2} \mathrm{O}_{5}(\mathrm{~F})\end{array}$ & $126,67 * *$ & $4,33 *$ & $32,36 * *$ & $41,22 * *$ & $113,89 * *$ & $0,07^{\text {ns }}$ & $17,92 * *$ & $0,16^{\mathrm{ns}}$ & $0,15^{\text {ns }}$ & $0,17^{\mathrm{ns}}$ \\
\hline $\begin{array}{l}\text { Doses de } \\
\mathrm{P}_{2} \mathrm{O}_{5} \text { (D) }\end{array}$ & $70,92 * *$ & $0,11^{\text {ns }}$ & $8,20 * *$ & $20,90 * *$ & $381,85^{* *}$ & $16,58 * *$ & $109,26 * *$ & $1,28 \mathrm{~ns}$ & $1,31^{\mathrm{ns}}$ & $0,17^{\text {ns }}$ \\
\hline Enxofre (E) & $2,79^{\mathrm{ns}}$ & $9,66 * *$ & $2,26^{\mathrm{ns}}$ & $0,49^{\text {ns }}$ & $15,91 * *$ & $1,57^{\text {ns }}$ & $0,25^{\mathrm{ns}}$ & $81,25 * *$ & $11,24 * *$ & 2,49 ns \\
\hline F x D & $2,75^{\mathrm{ns}}$ & $1,70^{\mathrm{ns}}$ & $1,90^{\mathrm{ns}}$ & $7,92 * *$ & $24,15^{* *}$ & $1,92^{\mathrm{ns}}$ & $2,00^{\mathrm{ns}}$ & $0,93^{\mathrm{ns}}$ & $0,14^{\mathrm{ns}}$ & $1,85^{\mathrm{ns}}$ \\
\hline $\mathrm{D} \times \mathrm{E}$ & $0,50^{\mathrm{ns}}$ & $0,95^{\mathrm{ns}}$ & $0,51^{\mathrm{ns}}$ & $5,87 * *$ & $3,69 *$ & $1,93^{\mathrm{ns}}$ & $0,70^{\text {ns }}$ & $0,59^{\text {ns }}$ & $0,87^{\text {ns }}$ & $0,37^{\text {ns }}$ \\
\hline $\mathrm{F} \times \mathrm{E}$ & $0,82^{\mathrm{ns}}$ & $1,84^{\mathrm{ns}}$ & $0,06^{\mathrm{ns}}$ & $0,00^{\mathrm{ns}}$ & $31,09 * *$ & $5,43^{*}$ & $3,81^{\text {ns }}$ & $0,81^{\mathrm{ns}}$ & $0,07^{\text {ns }}$ & $0,93^{\text {ns }}$ \\
\hline$D \times F \times E$ & $2,47^{\mathrm{ns}}$ & $3,59 *$ & $0,83^{\mathrm{ns}}$ & $0,60^{\mathrm{ns}}$ & $8,17 * *$ & $0,93^{\mathrm{ns}}$ & $0,85^{\text {ns }}$ & $2,87^{*}$ & $0,78^{\text {ns }}$ & $1,08^{\mathrm{ns}}$ \\
\hline $\begin{array}{l}\text { C.V. \% } \\
\end{array}$ & 3,84 & 6,46 & 7,82 & 6,39 & 16,5 & 56,80 & 20,48 & 34,16 & 43,25 & 3,80 \\
\hline
\end{tabular}

ns: não significativo $(\mathrm{P}>0,05),{ }^{*}$ e ${ }^{* *}$ significativo a $5 \%(\mathrm{P}<0,05)$ e $1 \%(\mathrm{P}<0,01)$, respectivamente.

dutividade, $\mathrm{pH}$ do solo e teores de $\mathrm{P}$ e $\mathrm{S}$ no solo na profundidade de 0-10 e $10-20 \mathrm{~cm}$. Houve diferença significativa entre as fontes de fósforo utilizadas, praticamente em todas as variáveis estudadas, com exceção dos teores de P e S na profundidade de $10-20 \mathrm{~cm}$ e pH do solo na profundidade de $0-10 \mathrm{~cm}$. Com relação à adição ou não de enxofre, ocorreram diferenças significativas apenas para teor de enxofre no tecido foliar, teor de P de $0-10 \mathrm{~cm}$ e teores de $\mathrm{S}$ nas profundidades de $0-10$ e $10-20 \mathrm{~cm}$.

Houve efeito significativo das fontes e de doses de fósforo utilizadas no teor de $\mathrm{P}$ no tecido foliar e teor de $\mathrm{P}$ nos grãos. Com aplicação de SFT, o teor médio de P (Tabela 3) foi de $2,7 \mathrm{~g} \mathrm{~kg}^{-1}$, ficando dentro da faixa adequada para a cultura do milho, enquanto, com o uso do FNR, o teor médio de $\mathrm{P}$ foi de $2,4 \mathrm{~g} \mathrm{~kg}^{-1}$, ficando, este, abaixo da faixa adequada para a cultura do milho, a qual segundo Malavolta et al. (1997), é de 2,5 a 3,5 $\mathrm{g} \mathrm{kg}^{-1}$. Entretanto, para Cantarella et al. (1997), a faixa de teor de P considerada adequada é de 2,0 a 4,0 $\mathrm{g} \mathrm{kg}^{-1}$. Essas diferenças entre as faixas adequadas de $\mathrm{P}$ no tecido foliar, observadas por estes autores, podem ser em decorrência do uso de dife- rentes cultivares, pois, Machado et al. (1999) observaram grande variabilidade entre genótipos e cultivares de milho para a eficiência do uso de fósforo.

Quanto ao efeito de doses de $\mathrm{P}_{2} \mathrm{O}_{5}$ utilizadas, ocorreu um acréscimo no teor de $\mathrm{P}$ foliar, ajustando-se aos dados o modelo quadrático com o maior teor foliar de $\mathrm{P}$, de $2,7 \mathrm{~g}$ $\mathrm{kg}^{-1}$ ocorrendo na dose estimada de $246 \mathrm{~kg} \mathrm{ha}^{-1}$ de $\mathrm{P}_{2} \mathrm{O}_{5}$ (Figura 1a). Para o teor de $\mathrm{P}$ no grão também ocorreu superioridade do SFT, que apresentou um teor médio de 2,15 $\mathrm{g} \mathrm{kg}^{-1}$, contra $1,94 \mathrm{~g} \mathrm{~kg}^{-1}$ para o FNR (Tabela 3), valores estes inferiores aos citados por Pauletti (2004), como valor adequado $3,8 \mathrm{~g} \mathrm{~kg}^{-1}$, obtido da média de quatro trabalhos. Observa-se que as doses de $\mathrm{P}_{2} \mathrm{O}_{5}$ utilizadas proporcionaram um incremento linear no teor de $\mathrm{P}$ no grão, com um acréscimo de $0,8 \mathrm{mg} \mathrm{kg}^{-1}$ para cada quilo de $\mathrm{P}_{2} \mathrm{O}_{5}$ aplicado (Figura 1b).

O enxofre no tecido foliar apresentou interação tripla significativa entre fontes, doses e enxofre. O enxofre adicionado juntamente com o FNR proporcionou aumento do teor no tecido vegetal até a dose de $250 \mathrm{~kg} \mathrm{ha}^{-1}$ de $\mathrm{P}_{2} \mathrm{O}_{5}$, com teor de $1,84 \mathrm{~g} \mathrm{~kg}^{-1}$, decrescendo com aumento das

Tabela 3. Medias dos teores de P foliar, S foliar, P grão, produtividade (Produt.), teores de P no solo extraídos por Mehlich-l (M-1) nas profundidades de 0-10 cm (P 10) e 10-20 cm (P 20), P no solo extraído por resina de troca aniônica na profundidade de 0-10cm (P 10 res.), teor de S disponível nas profundidades de 0-10 cm (S 10) e 10-20 cm (S 20), e o pH na profundidade de 0-10 cm em função da aplicação de fosfato natural reativo, superfosfato triplo, e adição ou não de enxofre elementar

\begin{tabular}{|c|c|c|c|c|c|c|c|c|c|c|}
\hline Adubação & P foliar & $S$ foliar & P grão & Produt. & $\begin{array}{l}\text { P } 10 \\
\text { M-I }\end{array}$ & $\begin{array}{l}\text { P } 20 \\
\text { M-I }\end{array}$ & P 10 res. & S 10 & S 20 & $\begin{array}{c}\mathrm{pH} \\
\mathrm{CaCl}_{2}\end{array}$ \\
\hline & - & $\mathrm{g} \mathrm{kg}^{-1}$ & 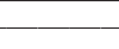 & $\mathrm{kg} \mathrm{ha}^{-1}$ & 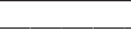 & - & $-\mathrm{mg} \mathrm{dm}^{-3}$ & & & \\
\hline FNR & $2,42 \mathrm{~b}$ & $1,74 \mathrm{~b}$ & $1,94 \mathrm{~b}$ & 8155 b & 36,49 b & 5,35 a & 59,16 b & 8,26 a & 5,13 a & $5,24 \mathrm{a}$ \\
\hline SFT & 2,69 a & 1,80 а & 2,15 a & 9013 a & 57,09 а & $5,56 \mathrm{a}$ & 73,53 а & 7,98 a & 5,34 a & $5,26 \mathrm{a}$ \\
\hline Com S & 2,57 a & $1,81 \mathrm{a}$ & $2,07 \mathrm{a}$ & 8692 a & 42,94 b & 5,95 a & 67,19 a & 11,25 a & 6,18 a & $5,21 \mathrm{a}$ \\
\hline Sem S & $2,53 \mathrm{a}$ & $1,72 \mathrm{~b}$ & 2,01 a & 8475 a & 50,64 a & 4,97 a & 65,15 a & $5,00 \mathrm{~b}$ & $4,29 \mathrm{~b}$ & 5,29 a \\
\hline C.V. \% & 3,84 & 6,46 & 7,82 & 6,39 & 16,5 & 56,80 & 20,48 & 34,16 & 43,25 & 3,80 \\
\hline
\end{tabular}

Letras iguais não diferem entre si pelo teste de tukey a $5 \%$. 

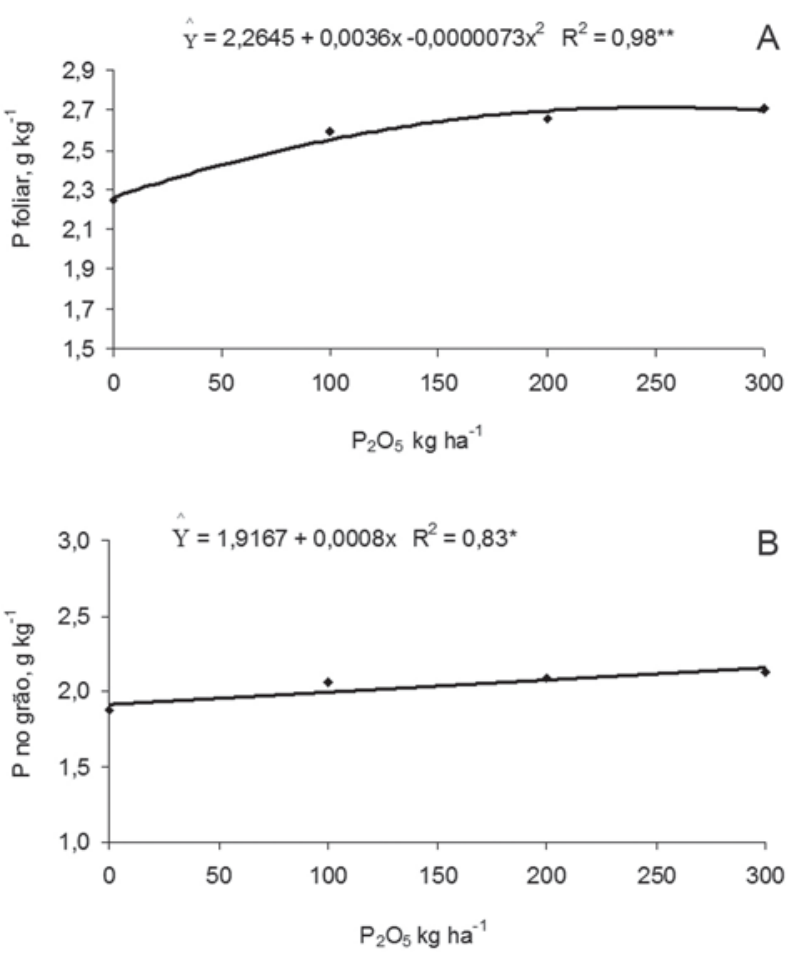

Figura 1. Teor de P no tecido foliar (a) e no grão (b) da cultura do milho em função das doses de fósforo aplicadas no solo, independente da adição ou não de enxofre.

doses (Figura 2a). Para o superfosfato triplo, ocorreu o inverso, ou seja, houve redução linear do teor de enxofre foliar com o aumento das doses de $\mathrm{P}_{2} \mathrm{O}_{5}$, uma vez que, a cada kg de $\mathrm{P}_{2} \mathrm{O}_{5}$ aplicado ocorreu um decréscimo de 0,0007 $\mathrm{g} \mathrm{kg}^{-1}$ (Figura 2b). Este decréscimo do teor de $\mathrm{S}$ foliar com o aumento das doses de SFT está, possivelmente, relacionado à maior solubilidade desta fonte, liberando mais $\mathrm{P}$ e estabelecendo competição com o enxofre liberado da solubilização do enxofre elementar.

Para as duas fontes de $\mathrm{P}_{2} \mathrm{O}_{5}$ utilizadas, o teor de enxofre no tecido foliar ficou dentro da faixa adequada para a cultura do milho, a qual, segundo Malavolta et al. (1997), é de 1,5 a 2,0 $\mathrm{g} \mathrm{kg}^{-1}$. Sem adição de enxofre, não houve diferença significativa no teor de enxofre foliar em função das doses de $\mathrm{P}_{2} \mathrm{O}_{5}$.

A produtividade de grãos apresentou uma resposta quadrática para as doses de fósforo utilizadas em ambas as fontes, sendo que as maiores produções foram obtidas com o uso do SFT, tendo como ponto de máximo rendimento $9791 \mathrm{~kg} \mathrm{ha}^{-1}$, na dose $247 \mathrm{~kg} \mathrm{ha}^{-1} \mathrm{de}_{2} \mathrm{O}_{5}$, enquanto que o rendimento máximo com o uso do FNR foi de 8522 $\mathrm{kg} \mathrm{ha}^{-1}$ na dose de $194 \mathrm{~kg} \mathrm{ha}^{-1}$ de $\mathrm{P}_{2} \mathrm{O}_{5}$ (Figura 3a).

Estes dados corroboram os encontrados por Kaminski \& Peruzzo (1997), que trabalhando com doses crescentes de $\mathrm{P}$ de diferentes fosfatos, em um solo com disponibilidade média de $\mathrm{P}$ e pH em água em torno de 5,5, encontraram incrementos na produção de grãos de milho com aumentos das doses de $\mathrm{P}_{2} \mathrm{O}_{5}$, onde os mais altos rendimentos foram

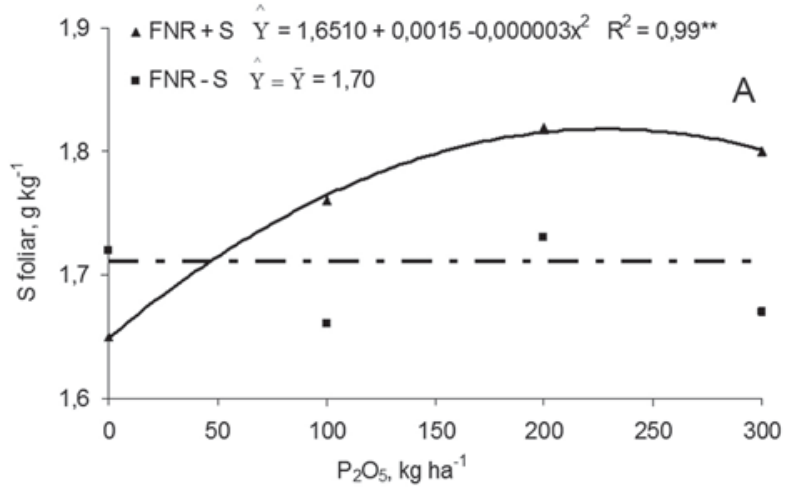
.

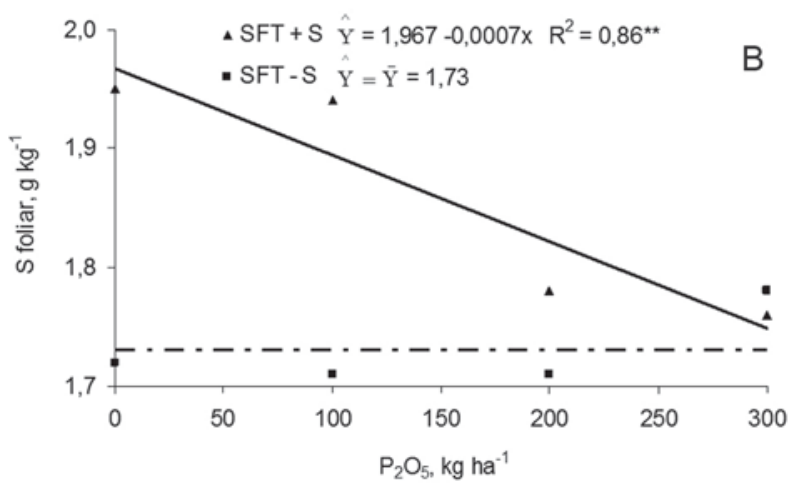

Figura 2. Teor de $\mathrm{S}$ no tecido foliar na cultura do milho em função da aplicação de FNR (a) e SFT (b) com adição ou não de S elementar.

obtidos com o uso de fontes solúveis de P no primeiro ano de cultivo. Isto indica que, em solos com acidez moderada e disponibilidade média a baixa de $\mathrm{P}$, os fosfatos solúveis aplicados no plantio são fontes mais adequadas de P, mas, segundo estes autores, em períodos mais longos há um efeito compensatório dos fosfatos naturais reativos, como destacado por Rein et al. (1994).

No presente trabalho, o teor de fósforo do solo no início do cultivo era baixo (2,9 $\left.\mathrm{mg} \mathrm{dm}^{-3}\right)$, o que justifica maior resposta da fonte de maior solubilidade. Barreto \& Fernandes (2002), em experimento a campo, com diferentes doses de $\mathrm{P}_{2} \mathrm{O}_{5}$ na forma de SFT, observaram incrementos na produção de grãos de milho até a dose de $155 \mathrm{~kg}$ ha ${ }^{-1}$ de $\mathrm{P}_{2} \mathrm{O}_{5}$, aplicado no sulco de plantio, apresentando também efeitos negativos na produtividade com a elevação das doses.

Esse efeito negativo na adubação localizada de fósforo sobre a produtividade tem sido atribuído, por alguns autores, à salinidade ou a toxidez, resultantes das altas concentrações dos adubos fosfatados, o que afetaria o crescimento das raízes (Peryea, 1990 citado por Prado et al., 2001).

Fazendo-se uma análise do índice de eficiência, considerando o aumento da produtividade em relação à testemunha, na dose de $194 \mathrm{~kg} \mathrm{ha}^{-1}$ de $\mathrm{P}_{2} \mathrm{O}_{5}$ para ambas as fontes, verifica-se que $\mathrm{o}$ aproveitamento do $\mathrm{P}$ aplicado foi melhor 

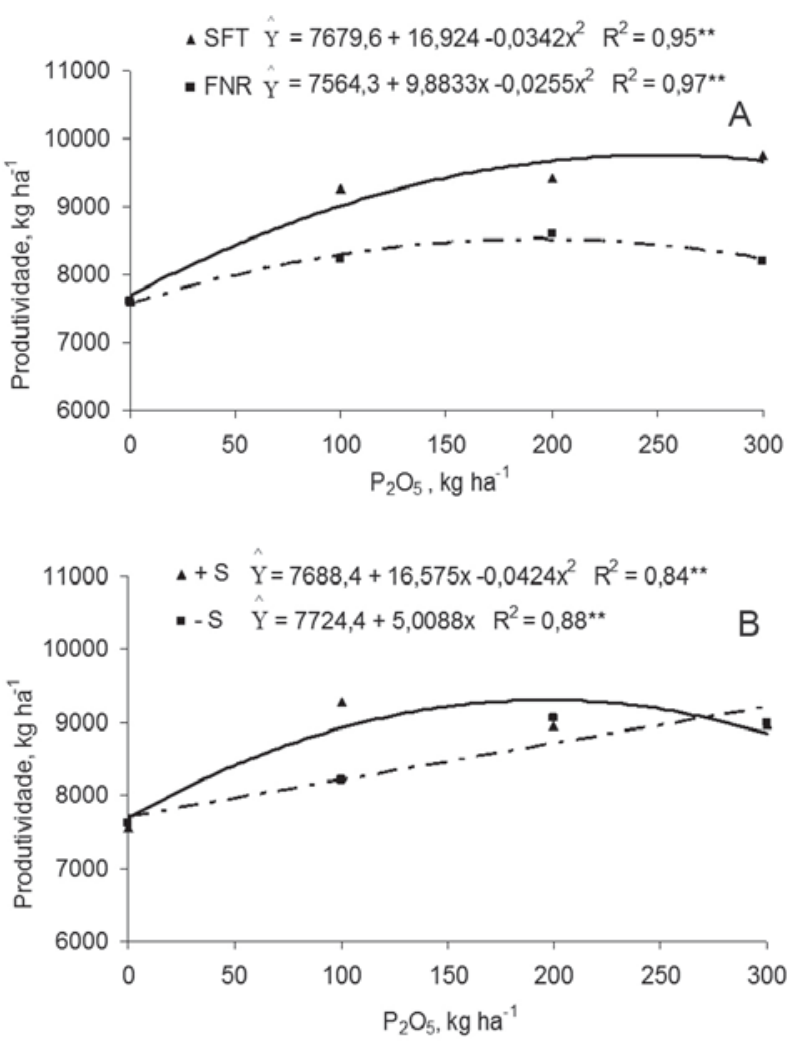

Figura 3. Produtividade de milho em função de fontes e doses de fósforo (a) e em função da adição ou não de enxofre elementar e doses de fósforo (b), para uma população de 60.000 plantas por ha.

para o SFT, produzindo $10,70 \mathrm{~kg} \mathrm{~kg}^{-1}$ de $\mathrm{P}_{2} \mathrm{O}_{5}$, enquanto, para o FNR, a produção foi de $4,85 \mathrm{~kg} \mathrm{~kg}^{-1} \mathrm{de}_{2} \mathrm{O}_{5}$.

Ao se analisar a relação custo/benefício, ou seja, o ganho em reais para cada real investido em ambas as fontes na dose de $194 \mathrm{~kg} \mathrm{ha}^{-1}$ de $\mathrm{P}_{2} \mathrm{O}_{5}$, com os preços médios praticados, na região, de R $\$ 380,00$ por tonelada de FNR (Gafsa), R \$ 740,00 por tonelada de SFT e de R $\$ 11,00$ por saca de $60 \mathrm{~kg}$ de milho (tomada de preço dia 07/08/06), obtém-se um retorno de $\mathrm{R} \$ 1,21$ para cada real investido na adubação com a fonte solúvel (SFT); com o uso do FNR este retorno foi de $\mathrm{R} \$ 0,65$, o que representa $47 \%$ a menos do que o obtido com o SFT. Com isso pode-se dizer que, no Latossolo Vermelho eutroférrico de textura argilosa com baixo teor de P, o uso do FNR como fonte de fósforo para a cultura do milho aplicado na linha de plantio é antieconômico no primeiro ano de plantio.

Para a produtividade, também houve interação significativa entre doses de $\mathrm{P}_{2} \mathrm{O}_{5}$ e aplicação de enxofre. Com a adição de enxofre, a máxima produção, $9308 \mathrm{~kg} \mathrm{ha}^{-1}$, ocorreu na dose de $195 \mathrm{~kg} \mathrm{ha}^{-1}$ de $\mathrm{P}_{2} \mathrm{O}_{5}$, enquanto, nos tratamentos sem enxofre a maior dose testada proporcionou uma produtividade de $9227 \mathrm{~kg} \mathrm{ha}^{-1}$ (Figura 3b). Com o exposto, observa-se que a adição de enxofre junto com a adubação fosfatada propiciou não apenas um maior incremento na produção de grãos, mas também uma melhor eficiência do adubo fosfatado, ou seja, com meno- res quantidades de adubos fosfatados obtiveram-se maiores rendimentos. Essa diferença de eficiência é melhor observada quando se faz a comparação considerando a produtividade máxima de grãos por kg de $\mathrm{P}_{2} \mathrm{O}_{5}$, pois, com adição de enxofre, foi de $47,7 \mathrm{~kg} \mathrm{~kg}^{-1}$ e, sem adição, foi de $30,8 \mathrm{~kg} \mathrm{~kg}^{-1}$.

No Brasil, são poucas as pesquisas sobre o uso de enxofre elementar como fonte de nutrientes para as plantas. Horowitz (2003) em trabalho realizado em casa de vegetação com quatro cultivos consecutivos de milho observou que a incorporação de enxofre elementar, na forma de pó, adicionado ao superfosfato triplo, apresentou eficiência agronômica crescente com o decorrer dos cultivos. Estes resultados indicam que a utilização do enxofre elementar associado a fontes de fósforo apresenta potencial de utilização.

Avaliando-se a eficiência do uso do fosfato (EUF) e o índice de eficiência agronômica (IEA), fica mais claro o efeito do enxofre sobre a disponibilidade de $\mathrm{P}$ para as plantas (Tabela 4). A maior eficiência de utilização do fertilizante para ambas as fontes foi obtida com a menor dose de $\mathrm{P}_{2} \mathrm{O}_{5}$ e adição de enxofre, apresentando valores de 11,20 $\mathrm{kg}$ de grãos de milho para cada kg de $\mathrm{P}_{2} \mathrm{O}_{5}$, com o uso do FNR aplicado ao solo, e de 23,02 kg para cada kg de $\mathrm{P}_{2} \mathrm{O}_{5}$, com o uso do SFT.

O índice de eficiência agronômica do FNR, usando como referência o SFT, apresentou maiores valores na dose de $200 \mathrm{~kg} \mathrm{ha}^{-1} \mathrm{de}_{2} \mathrm{O}_{5}$, tanto com a adição quanto com a não adição de enxofre, com valores de 52 e $57 \%$, respectivamente. Mas nota-se que, na dose de $100 \mathrm{~kg} \mathrm{ha}^{-1}$ de $\mathrm{P}_{2} \mathrm{O}_{5}$ com adição de enxofre, obteve-se índice de $49 \%$, valor este próximo aos encontrados com a dose de 200 kg. Estes índices estão próximos ao encontrado por Horowitz \& Meurer (2003), que encontraram IEA para o fosfato natural de Gafsa farelado de $42 \%$ em dois cultivos de milho em casa de vegetação. Já Piaia et al. (2002), trabalhando em casa de vegetação com a cultura da soja, encontraram IEA bem superior, com valor de $103 \%$ em solo com saturação de bases de $50 \%$, e de $77 \%$, em solo com saturação de bases de $65 \%$.

As concentrações médias de P no solo, na profundidade de 0-10 cm, após a colheita, diferiram significativamente, apresentando valores menores com o emprego do fosfato de Gafsa (FNR) em comparação com a utilização da fonte solúvel (SFT), para os dois extratores utilizados, com maior eficiência da resina em extrair fósforo do solo para as duas fontes (Tabela 3). Sarmento et al. (2002), em três semeaduras consecutivas de alfafa, utilizando como extrator a resina trocadora de íons, encontraram maiores concentrações para a fonte solúvel em relação ao fosfato natural, após o primeiro e segundo cultivo. No terceiro cultivo, houve superioridade do fosfato natural. Diante do exposto, com o passar do tempo, possivelmente haverá maior solubilização 
Tabela 4. Eficiência de utilização do fertilizante (EUF) e índice de eficiência agronômica (IEA) do fosfato natural reativo (FNR) para a produção de grãos de milho em função de doses de $\mathrm{P}_{2} \mathrm{O}_{5}$ aplicadas ao solo com adição ou não de enxofre elementar

\begin{tabular}{|c|c|c|c|c|c|c|c|}
\hline \multirow{2}{*}{$\begin{array}{l}\text { Doses } \\
\text { de } \mathrm{P}_{2} \mathrm{O}_{5}\end{array}$} & \multicolumn{3}{|c|}{ FNR } & \multicolumn{3}{|c|}{ SFT } & \multirow[b]{2}{*}{ IEA } \\
\hline & produção & $\begin{array}{l}\text { aumento } \\
\text { produção }\end{array}$ & EUF & produção & $\begin{array}{l}\text { aumento } \\
\text { produção }\end{array}$ & EUF & \\
\hline 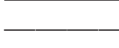 & $-\mathrm{kg} \mathrm{ha}^{-1}$ & 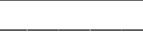 & $\mathrm{kg} \mathrm{kg}^{-1}$ & 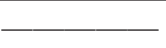 & $\mathrm{ha}^{-1}$ & $\mathrm{~kg} \mathrm{~kg}^{-1}$ & $\%$ \\
\hline \multicolumn{8}{|c|}{ Com enxofre } \\
\hline 0,0 & 7594 & - & - & 7545 & - & - & - \\
\hline 100 & 8711 & 1120 & 11,20 & 9847 & 2302 & 23,02 & 49 \\
\hline 200 & 8564 & 970 & 4,85 & 9418 & 1873 & 9,36 & 52 \\
\hline 300 & 8210 & 616 & 2,05 & 9727 & 2182 & 7,27 & 28 \\
\hline \multicolumn{8}{|c|}{ Sem enxofre } \\
\hline 0,0 & 7587 & - & - & 7654 & - & - & - \\
\hline 100 & 7729 & 142 & 1,42 & 8696 & 1042 & 10,42 & 14 \\
\hline 200 & 8634 & 1047 & 5,24 & 9496 & 1842 & 9,21 & 57 \\
\hline 300 & 8212 & 625 & 2,08 & 9799 & 2145 & 7,15 & 29 \\
\hline
\end{tabular}

do fósforo do fosfato natural, aumentando o teor de $\mathrm{P}$ no solo como descrito por Goedert \& Sousa (1984).

A concentração de fósforo no solo, extraída por resina de troca de íons, aumentou significativamente com o incremento das doses de fósforo para as duas fontes utilizadas, não diferindo quanto a adição ou não de enxofre (Figura 4c). Resultados semelhantes foram encontrados por Braga et al. (1991) e por Sarmento et al. (2002).

Para o extrator Mehlich-l, o qual apresenta ação preferencial na dissolução de fosfatos de cálcio, ocorreu interação significativa entre doses, fontes e enxofre (Figuras 4a, b). Para o FNR, as maiores concentrações de $\mathrm{P}$ no solo foram obtidas com a aplicação de enxofre, sendo obtida, para a dose de $300 \mathrm{~kg} \mathrm{ha}^{-1} \mathrm{de}_{2} \mathrm{O}_{5}$, a concentração de $88 \mathrm{mg} \mathrm{dm}^{-3}$ de $\mathrm{P}$ com a adição de $\mathrm{S}$, e, sem aplicação de $\mathrm{S}$, o teor foi de $68 \mathrm{mg} \mathrm{dm}^{-3}$ de P. Efeito contrário foi observado para o SFT, ocorrendo maior disponibilidade de $\mathrm{P}$ sem aplicação de $\mathrm{S}$, quando, com a dose de $300 \mathrm{~kg} \mathrm{ha}^{-1}$, obteve-se teor de $112 \mathrm{mg} \mathrm{dm}^{-3}$, enquanto, com adição de S, o teor foi de $81 \mathrm{mg} \mathrm{dm}^{-3}$. Stamford et al. (2004) obtiveram maior teor de $\mathrm{P}$ no solo extraído por Mehlich-1 no tratamento com fosfato natural e enxofre, apresentando valores superiores aos encontrados com a aplicação de SFT, verificando-se, assim, que houve atuação de microorganismos do solo sobre o S aplicado, disponibilizando P do FNR no solo.

A maior disponibilidade de fósforo do FNR com a adição de enxofre elementar a partir da dose de $100 \mathrm{~kg} \mathrm{ha}^{-1}$ de $\mathrm{P}_{2} \mathrm{O}_{5}$, possivelmente ocorreu em função da oxidação do enxofre por micro-organismos do solo, implicando em maior liberação de fósforo como observado por Stamford et al. (2004). Outra possibilidade seria a menor adsorção de fósforo no solo na presença de $\mathrm{S}_{-} \mathrm{SO}_{4}{ }^{2-}$, embora, de acordo com Parfitt (1978), na seqüência de preferência de adsorção, o sulfato estaria em quarto lugar, enquanto o fosfato é o ânion preferencialmente adsorvido.
$\mathrm{O} \mathrm{pH}$ do solo, determinado por $\mathrm{CaCl}_{2} 0,01 \mathrm{~mol} \mathrm{~L}^{-1}$, não foi alterado pelos tratamentos (Tabela 3). Este resultado provavelmente se deve a elevada capacidade tampão de acidez deste solo, em função da textura argilosa (694,5 $\mathrm{g} \mathrm{kg}^{-1}$ de argila). Santos (2002), utilizando tratamentos com fosfato natural, revestido com S inoculado com Acidithiobacillus, também não observou efeito de acidificação do solo, tendo em vista que o ácido sulfúrico produzido, provavelmente atuou na região próxima ao adubo, disponibilizando $\mathrm{P}$ do fosfato natural sem reduzir o pH do solo. Por outro lado, Stamford et al. (2004) observaram redução nos valores de $\mathrm{pH}$ do solo nos tratamentos com adição de $\mathrm{P}$, com uma variação de $\mathrm{pH}$ 6,2 para $\mathrm{pH} 4,8$, principalmente nos tratamentos com fosfato natural e enxofre.

Para teor de P no solo da camada de 0-10 cm, extraído com Mehlich-1, de amostras coletadas na linha de semeadura, houve interação significativa entre fontes e adição ou não de enxofre elementar, observando-se superioridade para o SFT em ambos os tratamentos com enxofre (Tabela 5). Nesta mesma profundidade, a aplicação de enxofre não influenciou significativamente o teor de $\mathrm{P}$ do solo quando se utilizou o FNR, ocorrendo o contrário para o SFT, o qual apresentou maiores valores de $\mathrm{P}$ nos tratamentos sem adição de enxofre.

O menor teor de P no solo, com a utilização de SFT e adição de enxofre elementar, provavelmente ocorreu devido a diminuição do $\mathrm{pH}$ ao redor do grânulo do adubo, pois a dissociação do SFT reduz o pH nesta região e, com isso, aumenta a adsorção de $\mathrm{P}$ e a formação de precipitados com $\mathrm{Fe}^{2+} \mathrm{e} \mathrm{Al}^{3+}$ (Figueiredo, 1985). Além disso, a oxidação do enxofre por micro-organismos do solo produz $\mathrm{H}_{2} \mathrm{SO}_{4}$, contribuindo ainda mais para a queda de $\mathrm{pH}$ e aumento da adsorção e formação de precipitados de P.

O maior teor de P no solo, encontrado com o uso de SFT na camada de 0-10 cm, difere dos resultados encon- 
Tabela 5. Teor de P no solo (mg dm³ ${ }^{-3}$ ) extraído por Mehlich-l nas camadas de 0-10 cm e 10-20 cm em amostras coletadas na linha de plantio, em função das fontes de fósforo aplicadas no solo e adição ou não de $S$

\begin{tabular}{lccc}
\hline Fonte de $\mathbf{P}_{2} \mathbf{O}_{5}$ & Com $\mathbf{S}$ & Sem $\mathbf{S}$ & Média \\
\cline { 2 - 4 } & \multicolumn{4}{c}{$\mathbf{0}-\mathbf{1 0} \mathbf{~ c m}$} \\
FNR dm & m \\
SFT & $38,03 \mathrm{Ab}$ & $34,96 \mathrm{Ab}$ & 36,49 \\
Média & $47,86 \mathrm{Ba}$ & $66,33 \mathrm{Aa}$ & 57,09 \\
\multicolumn{4}{c}{$\mathbf{1 0 - 2 0} \mathbf{c m}$} \\
FNR & 50,64 & 42,94 \\
SFT & $6,74 \mathrm{Aa}$ & $3,97 \mathrm{Ba}$ & 5,35 \\
Média & $5,15 \mathrm{Aa}$ & $5,98 \mathrm{Aa}$ & 5,56 \\
\hline
\end{tabular}

*Letras maiúsculas iguais na linha e minúsculas iguais na coluna, não diferem entre si pelo teste de Tukey a 5\%.
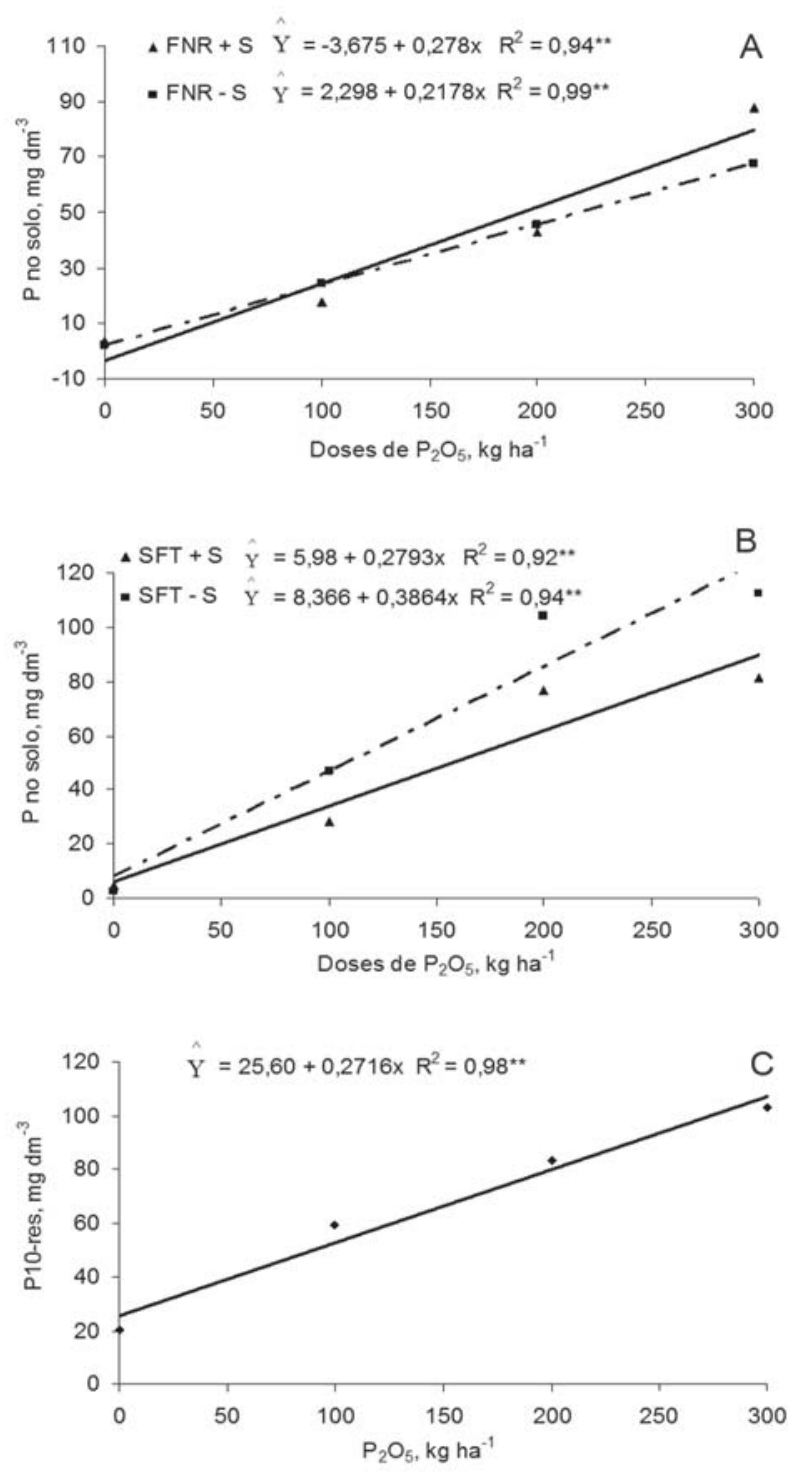

Figura 4. Teor de P no solo extraído por Mehlich-l na profundidade de 0-10 cm em função da aplicação de FNR com e sem adição de $S$ (a), aplicação de SFT com e sem adição de S (b) e teor de P no solo extraído por resina de troca aniônica na profundidade de 0-10 cm em função das doses de fósforo (c). trados por Stamford et al. (2004), que obtiveram teor de $\mathrm{P}$ no solo mais elevado com o uso de FNR e adição de enxofre, em comparação com o SFT, utilizando-se o extrator Mehlich-l, o que pode ser devido a diferenças quanto às propriedades físicas do solo utilizado. O teor de $\mathrm{P}$ no solo na profundidade de 10-20 cm não apresentou diferença significativa entre as fontes utilizadas, diferindo apenas quanto à adição ou não de enxofre para o FNR, uma vez que os maiores teores de $\mathrm{P}$ foram encontrados quando se adicionou enxofre (Tabela 5). Estes resultados indicam que pode ter ocorrido atuação de micro-organismos do solo sobre o enxofre elementar tornando o P mais disponível no solo.

\section{CONCLUSÕES}

O superfosfato triplo (SFT) proporciona maiores teores de P no tecido foliar e maior rendimento de grão em comparação com o fosfato natural reativo (FNR).

A adição de $30 \mathrm{~kg} \mathrm{ha}^{-1}$ de $\mathrm{S}^{\circ}$ não afeta o teor de $\mathrm{P}$ no tecido foliar.

A eficiência de uso do fertilizante é maior com a adição de enxofre e na menor dose de fósforo, $100 \mathrm{~kg} \mathrm{ha}^{-1} \mathrm{de}_{2} \mathrm{O}_{5}$ para ambas as fontes.

A adição de enxofre elementar proporciona maior índice de eficiência agronômica do FNR (43 \%), sendo que na ausência de enxofre o esse índice é de 33\%.

\section{REFERÊNCIAS}

Alvarez V VH, Dias LE, Ribeiro Jr ES, Souza RB \& Fonseca CA (2001) Métodos de análise de enxofre em solos e plantas. Viçosa, Editora UFV. 131p.

Barreto AC \& Fernandes MF (2002) Produtividade e absorção de fósforo por plantas de milho em função de doses e modos de aplicação de adubo fosfatado em solo de tabuleiro costeiro. Revista Brasileira de Ciência do Solo, 26:151-156.

Braga NR, Mascarenhas HAA, Bulisani EA, Raij B van, Feitosa CT \& Hirose R (1991), Eficiência agronômica de nove fosfatos em quatro cultivos consecutivos de soja. Revista Brasileira de Ciência do Solo, 15:315-319.

Cantarella H, Raij B van \& Camargo CEO (1997) Cereais. In: Raij B van, Cantarella H, Quaggio JÁ \& Furlani AMC (Eds.) Recomendação de adubação e calagem para o estado de São Paulo. $2^{\mathrm{a}}$ ed. Campinas, Instituto Agronômico de Campinas. p.45-47.

Defelipo BV \& Ribeiro AC (1981) Analise química do solo. Viçosa, Universidade Federal de Viçosa. 17p. (Boletim Extensão, 29).

EMBRAPA (1997) Centro Nacional de Pesquisa de Solos. Manual de métodos de análise de solos. $2^{\mathrm{a}}$ ed. Rio de Janeiro. 212p.

EMBRAPA (2006) Centro Nacional de Pesquisa de Solos. Sistema Brasileiro de Classificação de Solos. 2a ed. Brasília, Embrapa Produção de Informação. 306p.

Figueiredo OAR (1985) Reações de superfosfato triplo e de cama de galinha poedeira com um solo Latossolo Bruno. Dissertação de mestrado. Universidade Federal do Rio Grande do Sul, Porto Alegre, 85p.

Rev. Ceres, Viçosa, v. 57, n.5, p. 686-694, set/out, 2010 
Goedert WJ \& Lobato E (1984) Avaliação agronômica de fosfatos em solos de cerrado. Revista Brasileira de Ciência do Solo, 8:97-102.

Goedert WJ \& Sousa DMG (1984) Uso eficiente de fertilizantes fosfatados In: Simpósio Sobre Fertilizantes na Agricultura Brasileira. Anais, EMBRAPA, Brasília. p.255-289.

Horowitz N (2003) Oxidação e eficiência agronômica do enxofre elementar em solos do Brasil. Tese de Doutorado. Universidade Federal do Rio Grande do Sul, Porto Alegre, 111p.

Horowitz N \& Meurer EJ (2003) Eficiência de dois fosfatos naturais farelados em função do tamanho da partícula. Ciência Rural, 33:41-47.

Kaminski J \& Peruzzo G (1997) Eficácia de fosfatos naturais em sistemas de cultivo. Núcleo Regional Sul da Sociedade Brasileira de Ciência do Solo, Santa Maria, Boletim Técnico n.3, 31p.

Korndörfer GH (1999) Eficiência agronômica de fosfatos naturais reativos na cultura do milho. Scientia Agrícola, 56:1-9.

Machado CTT, Guerra JGM, Almeida DL \& Machado AT (1999) Variabilidade entre genótipos de milho para eficiência no uso de fósforo. Bragantia, 58:109-124.

Malavolta E, Vitti GC \& Oliveira AS (1997) Avaliação do estado nutricional das plantas: princípios e aplicações. $2^{\mathrm{a}}$ ed. Piracicaba, Associação Brasileira para a Pesquisa da Potassa e do Fosfato POTAFOS. 319p.

Mengel K \& Kirkby EA (1987) Principles of plant nutrition. 4 ed. Bern, International Potash Institute. 687p.

Ministério da Agricultura, Pecuária e Abastecimento, SISLEGIS (2007) Instrução normativa $N^{\circ} 5$ de 23 de fevereiro de 2007. Disponível em: < http://extranet.agricultura.gov.br/sislegis-consulta/servlet/VisualizarAnexo?id=14142>. Acessado em: 06 de junho de 2008.

Moreira A, Malavolta E \& Morais LAC (2002) Eficiência de fontes de fósforo na alfafa e centrosema cultivadas em Latossolo Amarelo. Pesquisa Agropecuária Brasileira, 10:1459-1466.

Parfitt RL (1978) Anion adsorption by soils and soil materials. Advances in Agronomy, 30:1-50.
Pauletti V (2004) Nutrientes: teores e interpretações. $2^{\mathrm{a}}$ ed. Castro, Fundação ABC. 86p.

Peruzzo G \& Wiethölter S (2002) Fosfato natural reativo deve ser usado no inverno? EMBRAPA, Empresa de Pesquisa Agropecuária Brasileira. Centro Nacional de Pesquisa de Trigo (CNPT), notícia 27 de 20/06/2002. Disponível em <http:// www.cnpt.embrapa.br/ > . Acessado em: 20 de novembro de 2006.

Piaia FL, Rezende PM, Furtini Neto AE, Fernandes LA \& Corrêa JB (2002) Eficiência da adubação fosfatada com diferentes fontes e saturação por bases na cultura da soja [Glycine max (L.) Merril]. Ciência e Agrotecnologia, 26:488-499.

Prado RM, Fernandes FM \& Roque CG (2001) Resposta da cultura do milho a modos de aplicação e doses de fósforo em adubação de manutenção. Revista Brasileira de Ciência do Solo, 25:8390 .

Rein TA, Sousa DMG \& Lobato E (1994) Eficiência agronômica do fosfato natural Carolina do Norte em solo do cerrado. Reunião Brasileira de Fertilidade do Solo e Nutrição de Plantas, 21. Petrolina, SBCS/EMBRAPA-CPATSA. p.38-40.

Santos KS (2002) Atuação de fosfato natural com adição de enxofre com Acidithiobacillus na solubilização de fósforo e no desenvolvimento de sabiá (Mimosa caesalpiniaefolia) em solo de tabuleiro. Dissertação de Mestrado. Universidade Federal Rural de Pernambuco, Recife, 68p.

Sarmento P, Corsi M \& Campos FP (2002) Eficiência do fosfato natural de Gafsa associado à calagem e gesso e sintomas nutricionais da alfafa, Medicago sativa L. Acta Scientiarum, 24:1155-1161.

Siddiqi MY \& Glass DM (1981) Utilization index: a modified approach to the estimation and comparison of nutrient utilization efficiency in plants. Journal Plant Nutrition, 4:289302 .

Stamford NP, Moura AMMF, Santos KS \& Santos PR (2004) Atuação de Acidithiobacillus na solubilização de fosfato natural em solo de tabuleiro cultivado com Jacatupé (Pachyrhizus erosus). Revista Brasileira de Ciência do Solo, 25:75-83. 